\title{
Die Folgen einer sportlich bedingten Gewichtsrestriktion im Kindesalter
}

\author{
Nathalie Boisseau \\ LAPHAP, Faculté des Sciences du Sport, Poitiers, Frankreich
}

\section{Schlüsselwörter}

Sport im Kindesalter - Gewichtsrestriktion - Wachstum, Kindesalter $\cdot$ Körperliche Reifung

\section{Zusammenfassung}

Junge Leistungssportler absolvieren heutzutage bereits von frühester Kindheit an ein körperlich anstrengendes Trainingsprogramm. Dieses intensive Training (Volumen und Intensität) hat einen gesteigerten Energieverbrauch zur Folge, den es durch eine höhere Nahrungsaufnahme auszugleichen gilt. Balletttänzer(innen), Eiskunstläufer(innen), Turner(innen) und rhythmische Sportgymnastinnen trainieren ab einem Alter von 5-6 Jahren, und zwar meist mehr als 20-30 Stunden pro Woche. In diesen Sportdisziplinen sowie in Sportarten mit Gewichtsklassen (Ringen, Judo, Boxen bzw. bei Jockeys) entscheiden sich die jungen Spitzensportler bewusst für eine Beschränkung der Nahrungsaufnahme, um sich ihren schlanken, vorpubertären Körperbau zu erhalten bzw. zum sogenannten «Gewichtmachen». Kinder im Wachstum müssen unbedingt ausreichend Energie und Mikronährstoffe aufnehmen, und eine Beschränkung der Kalorien- und Flüssigkeitszufuhr könnte bei trainierenden Kindern und Jugendlichen die Regulierung des Stoffwechsels und des Hormonhaushalts stören und sich somit auf das Wachstum, die Entwicklung und Körperzusammensetzung, den Menstruationszyklus und die Reproduktionsfähigkeit auswirken und das Risiko für Verletzungen, wie z.B. Ermüdungsbrüche, erhöhen. Ferner können diese Strategien, insbesondere bei heranwachsenden Mädchen, Essstörungen (z.B. Anorexie bzw. Bulimia nervosa) verursachen, das Körperbild und die Selbstwahrnehmung beeinflussen und zur Entstehung sozialer und emotionaler Fehlanpassungen führen. (c) 2006 Nestec Ltd., Vevey/S. Karger AG, Basel 0250-9652/06/0642-0079\$23.50/0

Fax +41613061234 E-Mail karger@karger.ch www.karger.com

\section{Einleitung}

Das schnelle Wachstum und die rasante körperliche Entwicklung in der Kindheit und Jugend führen zu einem höheren Bedarf an Energie und Mikronährstoffen. Dieser Bedarf ist bei jungen Sportlern aufgrund des höheren Ausmasses an Bewegung sogar noch höher. Bei jungen Leistungssportlern, insbesondere in ästhetischen Sportarten und Sportarten mit Gewichtsklassen, ist die Beschränkung der Nahrungs- und Flüssigkeitsaufnahme weit verbreitet und kann sich in der Wachstumsphase möglicherweise als gefährlich erweisen. Dieser Artikel berichtet über die möglicherweise nachteiligen gesundheitlichen Auswirkungen einer sportlich bedingten Gewichtsrestriktion im Kindesalter.

\section{Die Beschränkung der Nahrungs- und Flüssigkeitsaufnahme bei bestimmten Sportarten}

\section{Energie, Makro- und Mikronährstoffe}

Im Kindesalter und in der Jugend wirken sich das schnelle Wachstum und die rasante körperliche Entwicklung auf den Bedarf an Energie, Makro- und Mikronährstoffen aus.

\section{Energie}

Eine Schätzung des Energiebedarfs von Kindern, die Sport treiben oder in anderer Hinsicht körperlich sehr aktiv sind, ist aufgrund der starken intra- und interindividuellen Schwankungen relativ schwierig. Die vom Food and Nutrition Board berechnete Schätzung des Energiebedarfs beruht auf Gleichungen, die das Alter, die Körpergrösse, das Gewicht und den Aktivitätslevel einer Person, d.h. sitzend, mässig aktiv, aktiv oder sehr aktiv, berücksichtigen [1]. In der Altersgruppe der

Dr. Nathalie Boisseau

LAPHAP, Faculté des Sciences du Sport

4 allée Jean-Monnet

FR-86000 Poitiers (France)

Tel. +33 549454 042, Fax +33549453 396, E-Mail nathalie.boisseau@univ-poitiers.fr 
9- bis 13-Jährigen beträgt der Energiebedarf zwischen 1415 kcal/Tag bei einem 9-jährigen Mädchen mit sitzender Tätigkeit und $3038 \mathrm{kcal} / \mathrm{Tag}$ bei einem sehr aktiven 13-jährigen Jungen. Bei den 14- bis 18-Jährigen reicht der Energiebedarf von $1718 \mathrm{kcal} /$ Tag bei einem 14-jährigen Mädchen mit sitzender Tätigkeit bis zu $3804 \mathrm{kcal} /$ Tag bei einem sehr aktiven 18jährigen Jugendlichen. Der Gesamtenergieaufwand von Sportlern im Kindes- und Jugendalter ist also mit dem Grundumsatz, dem Wachstum und dem Mass an Bewegung verbunden. Bei Leistungssportlern beispielsweise erhöht sich durch das Sporttraining der Kalorienbedarf und bewirkt eine zusätzliche Energieaufnahme von 400-700 kcal/Tag [2, 3].

\section{Energiezufuhr bei Sportarten mit Gewichtsrestriktion}

Viele Leistungsturner(innen), rhythmische Sportgymnastinnen, Balletttänzer(innen) und Eiskunstläufer(innen) sind der Meinung, sie müssten Diät halten, um sich ihre schlanke Figur und ihren jugendlichen Körperbau zu bewahren. Bei Sportarten mit Gewichtsklassen (Ringen, Judo oder Boxen) beschränken viele Athleten während der Wettkampfsaison ihre Nahrungs- und Flüssigkeitsaufnahme, um bis zu drei Gewichtsklassen unterhalb ihres normalen Gewichts antreten zu können [4], weil sie glauben, sich dadurch einen Wettbewerbsvorteil gegenüber kleineren Gegnern zu verschaffen. Wiederholte Zyklen der Gewichtsabnahme und -zunahme sind somit die Regel, und es liegt eine regelmässige Störung der Energieaufnahme vor [5, 6]. Bei jungen Sportlern werden diese Gewichtsabnahmen und -zunahmen mit einer niedrigeren Stoffwechselrate und einem dadurch verringerten Energiebedarf assoziiert [7].

Wenngleich ihre Zahl begrenzt ist, so haben doch einige Studien belegt, dass die Energieaufnahme der Sportler während ihres gesamten Trainingprogramms (Turner(innen), rhythmische Sportgymnastinnen, Balletttänzer(innen), etc.), bzw. bei Sportarten mit Gewichtsklassen während der Wettkampfsaison, für ein gesundes Wachstum und das anstrengende Training theoretisch nicht ausreichend ist [8-20].

\section{Protein}

Um die für das Wachstum und insbesondere den Erhalt und die Entwicklung magerer Körpermasse, d.h. der Muskelmasse, erforderlichen essenziellen Aminosäuren bereitzustellen, bedarf es einer ausreichenden Proteinaufnahme. Der Proteinstoffwechsel spielt in der Tat eine wichtige Rolle bei der Errichtung und dem Remodeling der Muskelmasse. Bei erwachsenen Sportlern, die regelmässig ein anstrengendes Training absolvieren, ist der Tagesbedarf an Proteinen erhöht $[1,21]$. Bei Kindern und Jugendlichen (zwischen 9 und 18 Jahren) mit sitzendem oder mässig aktivem Lebensstil liegt der Proteinbedarf zwischen 0,73 und $0,85 \mathrm{~g} / \mathrm{kg}$ [1] bzw. 0,80 bis $1,0 \mathrm{~g} / \mathrm{kg}$ Körpermasse $[22,23]$. Diese Empfehlung bezieht sich jedoch nicht auf aktive bzw. sehr aktive Kinder. Tatsächlich gibt es derzeit keinerlei Informationen über den Proteinbedarf von jungen Sportlern. Die meisten Sportler essen spontan mehr, um ihre Energieaufnah- me zu steigern, und decken so ganz natürlich auch ihren Proteinbedarf. Bei Sportarten, die eine Restriktion der Nahrungsaufnahme zur Folge haben, kommt es unter Umständen zu einer Störung des Proteinstoffwechsels, die sich nachteilig auf das Wachstum bzw. die Gesamtmuskelmasse auswirkt.

Bei Turnerinnen und Balletttänzerinnen scheint die Proteinaufnahme auf den ersten Blick höher als 0,8-1,0 g/kg/Tag zu sein und in den meisten Fällen sogar bei 1,3-1,8 g/kg/Tag zu liegen, obwohl eine Bewertung der Ernährung dieser Sportlerinnen darauf hindeutet, dass viele dieser jungen Mädchen weniger Kalorien zu sich nehmen, als für ihr Alter und ihr Mass an Bewegung [17, 24-27] empfohlen wird [1]. Wie bereits in diversen Ernährungsstudien berichtet wurde, nehmen diese Sportlerinnen tatsächlich übermässig viel Eiweiss auf Kosten von Fett und Kohlenhydraten zu sich [27]. In einer jüngeren Studie konnte mit Hilfe des stabilen Isotops ${ }^{15} \mathrm{~N}$-Glycin nachgewiesen werden, dass sich das Training bei Turnerinnen vor und in der frühen Pubertät nicht auf den Proteinumsatz auswirkt [17].

Bei jungen Ringern und Judoka sieht die Sache ganz anders aus. Diverse Veröffentlichungen haben belegt, dass sich der Proteinstatus während der Gewichtsabnahme möglicherweise verändert [28] und unter Umständen vorübergehend zu einer negativen Stickstoffbilanz führt, die möglicherweise Wachstumsstörungen und Muskelverletzungen zur Folge hat. Friedlander et al. [29] wiesen vor kurzem darauf hin, dass eine dreiwöchige Restriktion der Kalorienaufnahme bei normalgewichtigen jungen Männern eine Änderung des Proteinstoffwechsels (Leucinumsatz und -oxidation) bewirkt.

\section{Kohlenhydrate}

Die empfohlene Kohlenhydrataufnahme basiert auf der Menge, die notwendig ist, um wichtige Gewebe (z.B. das Gehirn) mit Glukose zu versorgen, lässt dabei jedoch die Menge ausser Acht, die erforderlich ist, um die Glykogenspeicher in den Muskeln und in der Leber wieder aufzufüllen. Bei Erwachsenen ist die Leistung während des Ausdauertrainings bzw. bei wiederholtem sehr intensivem Training von den Glykogenspeichern abhängig, da es die Kohlenhydrate sind, die als limitierende Faktoren die Arbeitsleistung begrenzen [30, 31]. Bei präpubertären Kindern ist die glykolytische Kapazität angeblich nicht so effizient wie bei Jugendlichen oder Erwachsenen, eine Tatsache, die bei jungen Sportlern während des Ausdauertrainings eine höhere Fettverbrennung bewirkt [32]. Ob junge Leistungssportler von einer hohen Kohlenhydrataufnahme profitieren oder nicht, ist noch nicht endgültig geklärt. Die empfohlene Kohlenhydrataufnahme beträgt 50-60\% der Gesamtenergieaufnahme, wobei nur $10 \%$ aus einfachen Zuckern stammen sollten [33].

Turner(innen) oder Balletttänzer(innen) halten Kohlenhydrate für hochkalorische Nahrungsmittel. Obwohl die durchschnittliche Kohlenhydrataufnahme beispielsweise bei Turner(inne)n, Balletttänzer(inne)n und Eiskunstläufer(inne)n 
士50\% der Gesamtenergieaufnahme ausmacht, besteht letztere hauptsächlich aus einfachen Zuckern und nicht aus komplexen Kohlenhydraten [8, 17, 24, 27, 34]. Diese Ernährungsuntersuchungen zeigen, dass die Präferenz für Süsses sich nicht gut dafür eignet, die Glykogenspeicher in den Muskeln und in der Leber aufzufüllen. Bei Sportarten mit Gewichtsklassen führt die Beschränkung der Nahrungsaufnahme möglicherweise unmittelbar vor dem Wettkampf zu einem signifikanten Abbau der Glykogenspeicher in den Muskeln und in der Leber [5].

\section{Lipid}

Bezüglich der Gesamtfettaufnahme gibt es keine Richtlinien oder empfohlene tägliche Aufnahmemengen, aber es gibt Empfehlungen bezüglich der täglichen Aufnahmemengen an essenziellen Fettsäuren, wie z.B. Linol- und Linolensäure (n-3 und n-6; Tabelle 1).

Laut Empfehlungen sollte bei Kindern und Jugendlichen $30 \%$ der Gesamtenergie aus Fett stammen $[1,33]$. Obwohl Kinder bei Ausdauertraining verhältnismässig mehr Fett verbrennen als Erwachsene, gibt es keine Daten, die dafür sprechen würden, dass Kinder eine höhere Fettaufnahme benötigen.

Zur Gewichtskontrolle bzw. -restriktion wird in der Regel eine geringere Fettaufnahme empfohlen. Bei Leistungssportlern im Kindes- oder Jugendalter kann sich die Strategie, die Gesamtenergieaufnahme durch eine geringere Fettzufuhr zu reduzieren, möglicherweise als gefährlich erweisen. So ist in der Tat eine gewisse Mindestmenge an Körperfett erforderlich, um metabolische und hormonelle Anpassungen zu gewährleisten. Der zum Erhalt der Gesundheit und Fitness empfohlene Anteil an Körperfett beträgt bei Jungen 7\% und bei Mädchen 14\% [35]. Wird die Aufnahme fetthaltiger Nahrungsmittel reduziert oder sogar völlig eingestellt, so entspricht dies einem unnormalen Ernährungsverhalten, das einen Mangel an einfach und mehrfach ungesättigten Fetten zur Folge hat. Fettreiche Nahrungsmittel, wie z.B. Milchprodukte und rotes Fleisch, enthalten ferner qualitativ hochwertiges Protein, Kalzium, Magnesium, Eisen, Zink, Chrom, Vitamin $B_{12}$, fettlösliche Vitamine, etc., d.h. die für ein optimales Wachstum erforderlichen Nährstoffe. Alle Ernährungsuntersuchungen, die sich mit den ästhetischen Sportarten, u.a. Balletttanz, Eiskunstlauf, Turnen und rhythmischer Sportgymnastik befassen, weisen auf eine unzureichende Fettaufnahme hin $[8,11,17,24,27]$. Bei Sportarten mit Gewichtsklassen schwankt die Fettverteilung im Körper zwischen den Zeiten mit Gewichtsabnahme und Gewichtszunahme (JoJo-Diät) stark. Der Körperfettanteil ist folglich konstanten Änderungen unterworfen, ein Umstand, der sich während des Wachstums wahrscheinlich störend auf den Fettstoffwechsel auswirkt.

\section{Mikronährstoffe}

Eine unzureichende Energieaufnahme geht in der Regel mit einer sehr geringen Zufuhr an Mikronährstoffen (Mineralstoffen und Vitaminen) einher. In Studien, in denen junge
Tabelle 1. Eine adäquate Zufuhr von Linol- und Linolensäuren

\begin{tabular}{lll}
\hline Alter & \multicolumn{2}{l}{ Fettsäure, g/Tag } \\
\cline { 2 - 3 } & Linolsäure & Linolensäure \\
\hline $\begin{array}{l}\text { 9-13 Jahre } \\
\text { Jungen } \\
\quad \text { Mädchen }\end{array}$ & 12 & 1,2 \\
14-18 Jahre & 10 & 1,0 \\
$\quad$ Jungen im Jugendalter & 16 & 1,6 \\
$\quad$ Mädchen im Jugendalter & 11 & 1,1 \\
\hline
\end{tabular}

Sportler über eine Beschränkung der Kalorienzufuhr berichteten, erreichte die Mikronährstoffaufnahme tatsächlich nicht die empfohlene Höhe [5, 6, 8, 17, 24, 34, 36, 37]. Studien zur Nährstoffversorgung aktiver Kinder und Jugendlicher bzw. junger Sportler belegen in der Regel einen Mangel an zwei wichtigen Mikronährstoffen, nämlich Kalzium und Eisen. Diese Mikronährstoffe sind nicht nur für die Gesundheit, sondern auch zur Optimierung der sportlichen Leistung von entscheidender Bedeutung. Eisen ist in einer Reihe von Proteinen, u.a. Enzymen, sowie in Hämoglobin und Myoglobin enthalten, wobei Letzteres eine wichtige Rolle bei der Versorgung des gesamten Körpergewebes mit dem für den Stoffwechsel benötigten Sauerstoff spielt. Eisenmangel führt zu Anämie und beeinträchtigt die Leistungsfähigkeit. Auch Kalzium ist ein für die Entwicklung der Knochenmasse wichtiger Mikronährstoff - ein Prozess, der u.U.im Erwachsenenalter einen Schutz gegen Osteoporose bietet. Über 99\% des gesamten Kalziums im Körper befindet sich in den Zähnen und Knochen. Das restliche Kalzium verteilt sich auf das Blut, die extrazelluläre Flüssigkeit, die Muskeln und anderes Gewebe und ist dort an der Gefässkontraktion und -dilatation, der Muskelkontraktion, der Nervenübertragung und der Drüsensekretion beteiligt.

In der Pubertät steigt der Eisen- und Kalziumbedarf aufgrund der Erhöhung der Hämoglobinmasse, der verstärkten Gewebeablagerung, des Wachstumsschubs und des Einsetzens der Menstruation bei Frauen. Bei Sportarten, die mit einer Beschränkung der Energieaufnahme einhergehen, wird nur sehr wenig Kalzium oder Eisen mit der Nahrung aufgenommen [38, 39], da die jungen Sportler, entweder aus Sorge über ihre Fettaufnahme oder weil sie sich vegetarisch ernähren möchten, Milchprodukte und rotes Fleisch meiden.

\section{Flüssigkeit und Bewegung}

Der Hydratationsstatus vor der Bewegung bzw. dem Sport ist ein wichtiger Indikator für die Leistungsfähigkeit und Ausdauer und schränkt möglicherweise sowohl bei Erwachsenen als auch bei Kindern und Jugendlichen das Verletzungsrisiko ein. Laut Empfehlungen sollte man vor, während und nach dem Sport Flüssigkeit aufnehmen. Ein eventuelles Flüssig- 
keitsdefizit nach dem Sport sollte basierend auf dem Gewichtsverlust ausgeglichen werden.

Bei Sportarten mit Gewichtklassen gibt es nach wie vor nur wenige wissenschaftliche Daten zur Dehydratationsstrategie. Die in diesen Sportarten aktiven Athleten schränken jedoch aus praktischen Gründen vor dem Wettkampf die Aufnahme sämtlicher Flüssigkeiten (Wasser, Soda, Kaffee, Suppe etc.) ein und tragen darüber hinaus Unterwäsche aus Kunstfasern, um die Transpiration während des anstrengenden Trainings noch zu verstärken und so einen durch Flüssigkeitsverlust erzielten Gewichtsverlust zu fördern. Im Rahmen ihrer Versuche, das «richtige» Gewicht zu erzielen, experimentieren diese Sportler in der Regel auch mit anderen Strategien, wie z.B. Saunabesuchen, Diuretika und Erbrechen [5, 28]. Anscheinend erwarten sich die Sportler von diesen Strategien der Beschränkung der Flüssigkeitsaufnahme und akuten Dehydratation einen schnellen Gewichtsverlust, der ihnen die Teilnahme am Wettkampf in einer bestimmten Gewichtsklasse ermöglicht. Diese Strategien bleiben, wie nachfolgend dargestellt, nicht ohne Folgen.

\section{Die möglichen gesundheitlichen Folgen}

Die Schwierigkeit bei diesem Thema liegt im Nachweis der Kausalität zwischen einer Beschränkung der Nahrungs- und Flüssigkeitsaufnahme und den gesundheitlichen Folgen. Die Palette an Wechselwirkungen zwischen genetischen Faktoren und Umweltfaktoren (z.B. Ernährung) macht diesen Nachweis in der Tat schwierig. Weitere Studien tragen jedoch dazu bei, die potentiellen durch eine Gewichtsreduktion bei Leistungssportlern in der Kindheit oder Jugend hervorgerufenen negativen Auswirkungen auf die Gesundheit zu erklären.

\section{Wachstum und Entwicklung}

Obwohl bei Leistungsturner(inne)n, rhythmischen Sportgymnastinnen oder Balletttänzer(inne)n eine kleine Körpergrösse normal ist, und trotz der offensichtlichen gesundheitlichen Nutzwirkung der körperlichen Bewegung während des Wachstums, bestehen Bedenken, dass sich ein Trainingsprogramm auf hoher Leistungsebene bei Wettkampfsportlern in ästhetischen Sportarten möglicherweise nachteilig auf Wachstum und Entwicklung auswirkt. Wird das höhere Mass an Bewegung nicht durch ausreichende Ernährung ausgeglichen, so konkurriert der für das Training zu deckende Energiebedarf möglicherweise, insbesondere während des Wachstumsschubs in der Pubertät, mit dem Energiebedarf der zellulären Prozesse, die dem normalen Wachstum und der normalen Entwicklung zugrunde liegen, um die verfügbare Energie [40].

Turnerinnen haben beispielsweise einen geringeren Körperfettanteil als Kontrollpersonen und sind darüber hinaus signifikant kleiner und leichter als ihre Altersgenossen, wobei die Unterschiede bei älteren und fortgeschritteneren Turnerinnen ausgeprägter sind [41]. Verglichen mit ihren weniger sportlich aktiven Zwillingen oder anderen Geschwistern liegt bei den meisten Turnern und Turnerinnen eine Verzögerung der sexuellen Reifung vor. Längsschnittstudien zum Körperwachstum liefern zwar bessere Einblicke als Querschnittsdaten, aber auch sie weisen keinen kausalen Zusammenhang nach [42]. Die meisten dieser Studien jedoch lassen darauf schliessen, dass bei jungen Athletinnen, die ein anstrengendes Trainingsprogramm absolvieren, das Körperwachstum vermindert und die Entwicklung verzögert ist. Zahlreiche Daten deuten ferner darauf hin, dass nach Reduktion des Gesamttrainingumfangs bzw. bei ausreichender Ernährung [Einzelheiten siehe 42] ein Aufholwachstum stattfindet. Eine grosse Zahl von Berichten weist auf eine Beschränkung der Energieaufnahme bei jugendlichen Leistungssportlerinnen hin. Dies könnte eine Erklärung für die nachteiligen Auswirkungen auf das Wachstum und die Entwicklung sein, da der Wachstumsschub in der Pubertät empfindlich auf die Ernährungsfaktoren reagiert. Die zwischen einer Beschränkung der Nahrungsaufnahme und einem verminderten Wachstum bzw. einer verminderten körperlichen Reifung bei Kindern und Jugendlichen ablaufenden Mechanismen sind teilweise auf niedrigere Blutkonzentrationen der mit dem Wachstum verbundenen Hormone zurückzuführen. In der Tat wissen wir, dass eine chronische Beschränkung der Kalorien- und Proteinzufuhr die Konzentration des insulinähnlichen Wachstumsfaktors-1 (IGF-1) senkt [43]. Bei jungen Turnerinnen ist der IFG-1-Plasmaspiegel niedriger als bei Kontrollpersonen oder Schwimmern, ein Umstand, der mit einer unzureichenden Energieaufnahme, einer Verzögerung der körperlichen Reifung und einer Wachstumsunterbrechung in Verbindung gebracht wird [4446]. Bei männlichen Turnern dagegen lassen sich vor der $\mathrm{Pu}$ bertät und in der Frühpubertät keine niedrigeren IGF-1-Plasmakonzentrationen feststellen [47], es ist jedoch bekannt, dass eine solche Beschränkung der Nahrungsaufnahme bei Mädchen stets ausgeprägter ist.

\section{Körperzusammensetzung}

Die Körperzusammensetzung ist je nach Sportart verschieden. Bei Sportarten mit Gewichtsbeschränkung sind bezüglich des Körperfettanteils und der mageren Körpermasse, aufgrund der Gewichtsschwankungen in den Sportarten mit Gewichtsklassen, Unterschiede zwischen den ästhetischen Sportarten und den Sportarten mit Gewichtsklassen festzustellen.

\section{Körperzusammensetzung bei ästhetischen Sportarten}

Die meisten Athleten, die ästhetische Sportarten betreiben, bei denen das Aussehen eine wichtige Rolle für den Erfolg 
spielt, sind extrem dünn, da sie sich bewusst für eine Diät mit einer Beschränkung der Energieaufnahme entscheiden, um sich so ihren kindlichen Körperbau mit niedrigem Körpergewicht und einem niedrigen Körperfettanteil $\mathrm{zu}$ bewahren. Folglich wurde in der Fachliteratur umfangreich nachgewiesen, dass ein niedriger bis sehr niedriger Körperfettanteil bei Turner(inne)n, rhythmischen Sportgymnastinnen bzw. Balletttänzer(inne)n vor der Pubertät, in der Frühpubertät und im College-Alter kennzeichnend ist [Überblick siehe 48]. Paradoxerweise haben Studien an Athleten und Nichtathleten gezeigt, dass der Mensch auf eine Beschränkung der Energieaufnahme mit einer Senkung des Ruhestoffwechsels möglicherweise einhergehend mit einer gesteigerten Fettspeicherung reagiert [49]. Für die Athleten in diesen Sportarten wird es daher immer schwieriger, ihren Körperfettanteil niedrig zu halten, und nach Beendigung ihrer Wettkampfkarriere ist in der Regel eine Veränderung des Fettgewebes zu beobachten, die mit einer Zunahme der Grösse oder Zahl der Fettzellen einhergeht [15].

Ein Energieungleichgewicht hervorgerufen durch ein hohes Mass an Bewegung in Kombination mit einer Beschränkung der Nahrungsaufnahme wird im Allgemeinen mit einer geringeren Knochendichte assoziiert [49]. Sportler in ästhetischen Sportarten, wie z.B. Turnen, Eiskunstlauf, Ballett oder rhythmischer Sportgymnastik, haben jedoch, selbst bei einer reduzierten Kalziumzufuhr, eine signifikant höhere Knochenmineraldichte als Sportler in anderen Sportarten oder Kontrollpersonen, die keinen Sport treiben [48]. Zu der höheren Knochenmineraldichte tragen sowohl die Gene als auch die Belastung der Knochen (wiederholtes Springen und Landen) bei. Der Knochenmineralgehalt und sogar die Knochengrösse sind also bei Spitzensportlern, die sogenannte "very high impact activities» (Sportarten mit sehr hoher Sprungbelastung) ausüben, unabhängig von ihrer Nahrungsaufnahme erhöht.

\section{Körperzusammensetzung bei Sportarten mit}

Gewichtsklassen

Bei Sportarten mit Gewichtsklassen, wie z.B. Ringen, Judo etc., kommt es zu schnellen, häufigen und starken Gewichtsschwankungen. Die Körperzusammensetzung ist somit möglicherweise regelmässigen Änderungen unterworfen. Bei kurzfristigen Gewichtsreduktionen werden hauptsächlich Wasser, Fett, Eiweiss und Glykogen abgebaut. Bei der Gewichtsabnahme und dem Abbau der Fettspeicher ändert sich unter Umständen die Körperfettverteilung. Je länger die Energieaufnahme beschränkt wird, desto stärker sind die Fettspeicher am Gesamtgewichtsverlust beteiligt. In den meisten Fällen jedoch machen die jungen Sportler eine Blitzdiät mit einer kurzfristigen Gewichtsabnahme, die einen beträchtlichen Verlust an magerer Körpermasse und Wasser zur Folge hat [5, 15]. Wird die Energieaufnahme weniger stark, dafür aber über einen längeren Zeitraum beschränkt, wird der Abbau an magerer Muskelmasse minimiert und der Fettverlust maximiert.
Nimmt ein Sportler wiederholt Gewicht ab und wieder zu, dann werden unter Umständen die Fettspeicher abgebaut und in anderen Körperregionen wieder aufgebaut [15]. In diesem Fall ändert sich möglicherweise die Fettverteilung, auch wenn der Gesamtfettanteil gleich bleibt. Über die typische Gewichtszunahme bei ehemaligen Sportlern mit Gewichtsklassen ist bisher wenig bekannt, aber die JoJo-Diät könnte später ein erhöhtes Taille-Hüfte-Verhältnis zur Folge haben, das wie bei adipösen Menschen auf ein erhöhtes Risiko für kardiovaskuläre Erkrankungen schliessen lässt.

\section{Nahrungsverwertung}

Die Nahrungsverwertung kann als das Verhältnis von Gewichtsänderung zu Kalorienaufnahme definiert werden. Sie ist ein Index, wie viel eine Person zur Erhaltung eines bestimmten Gewichts oder einer bestimmten Körperzusammensetzung essen muss. Je besser die Nahrungsverwertung, desto weniger Kalorien müssen aufgenommen werden, um 1 kg Körpergewicht zu erhalten. Ausgehend von verschiedenen Studien zur Nahrungsaufnahme und Körperzusammensetzung junger Sportler und Sportlerinnen vermuteten Brownell et al. [15], dass bei Sportlern mit niedrigem oder schwankendem Gewicht die Nahrungsverwertung unter Umständen verbessert wird. Bei diesem Mechanismus handelt es sich wahrscheinlich um eine physiologische Anpassungsreaktion, die den lebenswichtigen Prozess der Energiespeicherung erleichtert. Bei einer Erhöhung der Kalorienzufuhr könnte es jedoch zu einer schnellen Gewichtszunahme kommen. Laut anekdotischen Berichten beklagen sich manche Turnerinnen, dass die Wahrung ihres niedrigen Gewichts zunehmend schwieriger werde und sie chronisch mit ihrem Gewicht zu kämpfen hätten.

\section{Menstruationsstörungen und Fortpflanzungsfähigkeit}

Dixon et al. [50] behaupteten, ein hohes Mass an Bewegung führe zu einer erhöhten Endorphinausschüttung, die in Kombination mit einer Beschränkung der Kalorienzufuhr eine gestörte Regulation der Hypothalamusaktivität zur Folge habe. Eine gestörte Regulation des Hypothalamus löst ein Absinken der pulsatilen Frequenz der Gonadotropinsekretion aus (follikelstimulierendes Hormon, luteinisierendes Hormon) und führt häufig zu einer verzögerten pubertären Reifung und folglich zu Oligo- oder Amenorrhoe [8]. Bezüglich der Regulation der Hypothalamus-Hypophyse bestehen zwischen den Sportarten grosse Unterschiede. Es ist allgemein bekannt, dass Spitzenturnerinnen aufgrund der durch Diät und Trainingsprogramm verursachten hormonellen Änderungen zur verzögerten Menarche und zu Regulationsstörungen der Menstruation neigen (Amenorrhoe, Oligomenorrhoe, Anovulation, Fehlen der lutealen Phase) [51]. Die Amenorrhoe ist zwar die 
extremste Form, aber auch andere Ausprägungen der Menstruationsstörung können die Östrogenspiegel unterdrücken und sich so auf die Knochengesundheit und Fertilität auswirken. Mädchen im Kindes- und Jugendalter, die ästhetische Sportarten betreiben, können somit theoretisch einen Symptomkomplex entwickeln, der als «Sportlerinnentriade» bezeichnet wird und aus drei Komponenten besteht: Essstörung, Amenorrhoe und Osteoporose (selbst wenn diese dritte Komponente, wie oben erläutert, bei den sogenannten «high-impact activities» eher seltener auftritt).

Parallel zu den Menstruationsstörungen bei Frauen ist bei Männern unter Umständen der Testosteronspiegel unterdrückt oder gesenkt. Strauss et al. [52] ermittelten im Blutserum von männlichen Ringern, insbesondere bei einem Körperfettanteil von unter 5\%, niedrige Testosteronkonzentrationen. Nach dem Ende der Wettkampfsaison normalisierten sich mit der Gewichtszunahme die Testosteronkonzentrationen wieder. Ein geringes Körpergewicht, ein niedriger Körperfettanteil, Mangelernährung und ein hohes Mass an Training bedeuten bei männlichen Athleten unter Umständen eine Gefahr für die Zeugungsfähigkeit.

\section{Eisenstatus und Anämie}

Neben niedrigen Hämatokrit- und Ferritinwerten $(<20 \mu \mathrm{g} /$ 1) werden bei trainierten Sportlern häufig auch niedrige Hämoglobinkonzentrationen im Blut (13-14 g/100 ml bei Männern und $12 \mathrm{~g} / 100 \mathrm{ml}$ bei Frauen) beobachtet. Diese Parameter definieren den Begriff «Sportanämie» [53]. In der Pubertät erhöht sich die Hämoglobinmasse. Aus diesem Grund und wegen der Gewebeablagerung, des Wachstumsschubs und des Einsetzens der Menstruation bei Frauen ist der Eisenbedarf in der Pubertät erhöht [33]. Gründe für niedrige Eisenwerte können eine mechanische Hämolyse, Darmblutungen, Hämaturie, Schwitzen, eine niedrige Eisenzufuhr und eine schlechte Eisenabsorption im Darm sein [53]. Die resultierende Abnahme des Gastransports im Blut und der Aktivität der Muskelenzyme stört die Leistung. Mit dem Eisenstatus von jugendlichen Sportlern haben sich bisher nur wenige Studien befasst, aber es scheint, dass diese Sportler auch als Erwachsene anfällig für einen nichtanämischen Eisenmangel sind [54, 55]. Andererseits aber werden bei Turnern und Turnerinnen leere Eisenspeicher (niedrige Ferritinkonzentrationen) nachgewiesen [38].

\section{Verletzungen}

Mit der Untersuchung von Verletzungen bei jungen Spitzensportlern haben sich viele Studien befasst. Retrospektive und prospektive Studien belegen hohe, eindeutig mit dem Leistungsniveau verbundene Verletzungsraten bei Turnerinnen. Akute Verletzungen (z.B. Ermüdungsbrüche und Bänder- verletzungen) an den Füssen und Zehen treten ebenso wie Knöchelverletzungen häufig auf [48]. Einige dieser akuten Verletzungen könnten auf eine Beschränkung der Nahrungszufuhr zurückzuführen sein, da allgemein bekannt ist, dass eine niedrige Östrogenkonzentration sowie eine unzureichende Protein- und Kalziumaufnahme kombiniert mit einer späten Menarche möglicherweise eine höhere Zahl an Ermüdungsbrüchen zur Folge hat [56].

Bei Sportarten mit Gewichtsklassen werden während der Gewichtsabnahme vor dem Wettkampf aufgrund der Beschränkung der Flüssigkeitsaufnahme häufig Muskelskelettverletzungen beobachtet [35]. Wird der Wettkampf in einem stark geheizten Umfeld abgehalten, so besteht darüber hinaus die Möglichkeit einer Hitzeverletzung.

\section{Die Auswirkungen der Dehydratation}

Eine starke Gewichtsreduktion könnte sich tatsächlich störend auf die physiologischen Funktionen auswirken. So könnte die Dehydratation beispielsweise ein Sinken des renalen Plasmaflusses und der Filtration zur Folge haben und sich somit nachteilig auf die Nieren auswirken. Junge Ringer berichten ferner über Gereiztheit, Verstopfung und ein «Gefühl der Abgeschlagenheit» [37]. Wie sich eine schnelle Gewichtsabnahme und Dehydratation auf die physiologische Leistung auswirkt, ist hinreichend dokumentiert [35]. Zu den durch dieses Vorgehen verursachten Folgen zählen: die Abnahme der Muskelkraft und der anaeroben Ausdauerleistung; ein erhöhter Ruhepuls und ein niedrigerer Maximalpuls; ein niedrigeres Herzschlagvolumen und dadurch eine geringere Fähigkeit, eine konstante Leistung aufrechtzuerhalten; ein geringerer Sauerstoffverbrauch; eine Störung der mit der Wärmeregulierung verbundenen Prozesse; ein geringeres Plasma- und Blutvolumen; ein Abbau des in den Muskeln und möglicherweise auch des in der Leber gespeicherten Glykogens; eine Störung der GlukoseHomöostase; ein veränderter Hormonstatus; eine herabgesetzte Immunfunktion, etc. [35]. In vitro hat sich ferner ergeben, dass die Dehydratation der Zellen eventuell zur Einleitung der Protein- und Glykogenaufspaltung führt, durch welche die zelluläre Osmolarität als Reaktion auf das gesenkte intrazelluläre Flüssigkeitsvolumen angepasst werden soll [57].

\section{Essstörungen}

Von Essstörungen spricht man, wenn die Nahrungsaufnahme oder die Unterlassung der Nahrungsaufnahme als Reaktion auf ein äusseres anstatt eines inneren Signals erfolgt. Essstörungen bei Sportlerinnen rücken zunehmend in den Mittelpunkt des Interesses. Athleten, die ästhetische Sportarten, bei denen eine schlanke Figur notwendig ist, oder Sportarten mit Gewichtsklassen betreiben, sind anfälliger für Ess- 
störungen als andere Sportler [58]. Rhythmischen Sportgymnastinnen, Turner(inne)n, Balletttänzer(inne)n und Eiskunstläufer(inne)n, die Wettkämpfe bestreiten, wird beispielsweise vorgeworfen, eine unphysiologische Gewichtsabnahme zu befürworten, die sich zu einer manifesten Anorexie bzw. Bulimia nervosa auswachsen kann [58]. Eine der Erklärungen hierfür ist unter Umständen der enorme Erfolgsdruck und der Druck, ein ideales Körpergewicht vorzuweisen, der auf den jungen Athleten lastet. So berichten Athleten bzw. Athletinnen, die ästhetische Sportarten betreiben, über eine grössere Unzufriedenheit mit ihrem Körper, eine niedrigere Selbstachtung und eine anhaltendere Beschränkung der Nahrungsaufnahme als andere Sportler. Sind diese Sportler nicht in der Lage, ihr Gewicht und ihren Körperbau zu kontrollieren, so kann dies zu Frustration, Schuldgefühlen und sogar Verzweiflung führen, die soziale und emotionale Störungen zur Folge haben können.

\section{Morbidität und Mortalität}

Die Ernährungspraktiken mancher Athleten führen unter Umständen zu einem höheren Risiko für akute oder chronische Krankheiten. Von der spezifischen Qualität der Ernährung (gesättigte Fettsäuren, einfache Zucker, Natrium, etc.) einmal abgesehen, wirkt sich möglicherweise auch das Körpergewicht an sich gesundheitlich aus. Es ist allgemein bekannt, dass eine chronische Beschränkung der Kalorienaufnahme das Risiko für bestimmte Krebsarten [59] und Krankheiten, wie Koronarerkrankungen, Bluthochdruck und Diabetes, senkt. Bei Athleten mit einem niedrigen Körpergewicht und Essstörungen liegt jedoch möglicherweise ein Mangel an wichtigen Nährstoffen (z.B. Vitaminen, Mineralstoffen, essenziellen Aminosäuren, essenziellen Fettsäuren) vor, der möglicherweise die metabolischen und organischen Funktio- nen verändert. So ist beispielsweise bei Patienten, die unter einer Anorexie nervosa leiden, aufgrund der sehr niedrigen Kaliumspiegel die QT-Dispersion grösser als bei veranlagungsbedingt dünnen und normalgewichtigen Frauen (die QT-Dispersion wurde definiert als die Differenz zwischen dem längsten und dem kürzesten QT-Intervall beim 12-KanalEKG) [60]. Auch die wiederholte Gewichtsabnahme und -zunahme kann diverse negative gesundheitliche Auswirkungen zur Folge haben. So wird in der Tat die Gewichtsabnahme mit einer Blutdrucksenkung und die Gewichtszunahme mit einer Blutdruckerhöhung in Verbindung gebracht. Da die Zeit der Gewichtszunahme wichtiger ist als die der Gewichtsabnahme, könnte sich dies auf den Blutdruck auswirken. Diverse Studien behaupten ferner, dass die renale Ischämie bei Ringern möglicherweise eine Folge der wiederholten Gewichtsabnahme und -zunahme ist und möglicherweise mit einer Prädisposition für einen späteren Bluthochdruck einhergeht [15].

\section{Schlussfolgerung}

Dieser Übersichtsartikel belegt, dass eine sportlich bedingte Gewichtsrestriktion im Kindes- und Jugendalter zahlreiche, manchmal irreversible gesundheitliche Folgen hat. Zur Vermeidung dieser weitreichenden Folgen empfehlen wir, unabhängig vom Alter und Geschlecht der Sportler, eine sorgfältige Überwachung des Ernährungsstatus von jungen Leistungssportlern, insbesondere von Leistungssportlern in Sportarten, die mit einer beschränkten (ästhetische Sportarten) oder unregelmässigen (Sportarten mit Gewichtsklassen) Nahrungsaufnahme einhergehen. Ein psychologischer Ansatz und die Aufklärung von Sportlern, Trainern und Eltern hinsichtlich ernährungsbezogener Themen sollten die von einem Fachmann oder Ernährungswissenschaftler für Sporternährung unterbreiteten Ernährungsempfehlungen abrunden.

\section{Literatur}

1 DRI: Dietary Reference Intakes for Energy, Carbohydrate, Fiber, Fat, Fatty Acids, Cholesterol, Protein, and Amino Acids (Macronutrients). Washington, National Academy Press, 2002.

2 Tveit-Milligan P, Spindler AA, Nichols JE: Genes and gymnastics: a case study of triplets. Sports Med Training Rehab 1993;4:4752.

3 Lindholm C, Hagenfeldt K, Ringertz BM: Pubertal development in elite juvenile gymnasts. Effects of physical training. Acta Obstet Gynecol Scand 1994;73:269-273.

-4 Steen SN, Brownell KD: Patterns of weight loss and regain in wrestlers: has the tradition changed? Med Sci Sports Exerc 1990;22:762768

Gewichtsrestriktion bei jungen Sportlern und gesundheitliche Folgen
${ }_{5}$ Boisseau N, Vera-Perez S, Poortmans JR: Food and fluid intake in adolescent female judo athletes before competition. Pediatr Exerc Sci 2005;17:62-71.

-6 Oppliger RA, Steen SA, Scott JR: Weight loss practices of college wrestlers. Int J Sport Nutr Exerc Metab 2003;13:29-46.

7 Steen SN, Oppliger RA, Brownell KD: Metabolic effects of repeated weight loss and regain in adolescent wrestlers. JAMA 1988; 260:47-50

$\checkmark 8$ Weimann E, Witzel C, Schwidergall S, Bohles HJ: Peripubertal perturbations in elite gymnasts caused by sport specific training regimes and inadequate nutritional intake. Int J Sports Med 2000;21:210-215.
9 Theintz GE, Howald H, Allemann Y, Sizonenko PC: Growth and pubertal development of young female gymnasts and swimmers: a correlation with parental data. Int J Sports Med 1989;10:87-91.

10 Schwidergall S, Weimann E, Witzel C, Molenkamp G, Brehl S, Bohles H: Nutritional behaviour of female and male high performance gymnasts. Wien Med Wochenschr 1988;148:243-244.

11 Lindholm C, Hagenfeldt K, Hagman U: A nutrition study in juvenile elite gymnasts. Acta Paediatr 1995;84:273-277.

12 Kiningham RB, Gorenflo DW: Weight loss methods of high school wrestlers. Med Sci Sports Exerc 2001;33:810-813. 
13 Gurd B, Klentrou P: Physical and pubertal development in young male gymnasts. J Appl Physiol 2003;95:1011-1015.

14 Georgopoulos N, Markou K, Theodoropoulou A, Paraskevopoulou P, Varaki L: Growth and pubertal development in elite female rhythmic gymnasts. J Clin Endocrinol Metab 1999;84:4525-4530.

-15 Brownell KD, Steen SN, Wilmore JH: Weight regulation practices in athletes: analysis of metabolic and health effects. Med Sci Sports Exerc 1987; 19:546-556.

- 16 Baxter-Jones AD, Helms P, Maffulli N, Baines-Preece JC, Preece M: Growth and development of male gymnasts, swimmers, soccer and tennis players: a longitudinal study. Ann Hum Biol 1995;22:381-394.

- 17 Boisseau N, Persaud C, Jackson AA, Poortmans JR: Training does not affect protein turnover in pre- and early pubertal female gymnasts. Eur J Appl Physiol 2005;94:262267.

18 Fogelholm GM, Kukkonen-Harjula TK, Taipale SA, Sievänen HT, Oja P, Vuori IM: Resting metabolic rate and energy intake in female gymnasts, figure-skaters and soccer players. Int J Sports Med 1995;16:551-556.

19 Bass S, Bradney M, Pearce G, Hendrich E, Inge K: Short stature and delayed puberty in gymnasts: influence of selection bias on leg length and the duration of training on trunk length. J Pediatr 2000;136:149-155.

20 Zonderland ML, Claessens AL, Lefevre J, Philippaerts R, Thomis M: Delayed growth and decreased energy intake in female gymnasts; in Armstrong N, Kirby B, Welsman J (eds): Children and Exercise XIX: Promoting Health and Well-Being: Proceedings of the XIXth International Symposium of the European Group of Pediatric Work Physiology. London, E\&FN Spon, 1997, pp 553-556.

21 Poortmans JR: Protein metabolism; in Poortmans JR (ed): Principles of Exercise Biochemistry. Basel, Karger, 2004, pp 227278.

22 FAO/WHO: Joint Expert Committee: Energy and Protein Requirements. Geneva, World Health Organization, 1986.

23 Martin A: Apports nutritionnels conseillés pour la population française. Paris, Tec \& Doc, 2001.

-24 Cupisti A, D’Alessandro S, Castrogiovanni $S$, Barale E, Morelli E: Nutrition survey in elite rhythmic gymnasts. J Sports Med Phys Fit 2000;40:350-355

25 Weimann E, Blum WF, Witzel C, Schwidergall S, Bohles HJ: Hypoleptinemia in female and male elite gymnasts. Eur J Clin Invest 1999;29:853-860

-26 Fogelholm GM, Kukkonen-Harjula TK, Taipale SA, Sievanen HT, Oja P, Vuori IM: Resting metabolic rate and energy intake in female gymnasts, figure-skaters and soccer players. Int J Sports Med 1995;16:551-556.

-27 Benson J, Gillien DM, Bourdet K, Loosli AR: Inadequate nutrition and chronic calorie restriction in adolescent ballerinas. Phys Sportmed 1985;13:79-90.
28 Horswill CA, Park SH, Roemmich JN: Changes in the protein nutritional status of adolescent wrestlers. Med Sci Sports Exerc 1990;22:599-604.

29 Friedlander AL, Braun B, Pollack M, MacDonald JR, Fulco CS: Three weeks of caloric restriction alters protein metabolism in normal-weight, young men. Am J Physiol Endocrinol Metab 2005;289:E446-E455.

30 Coyle EF, Coyle E: Rating carbohydrates that speed recovery from training. Phys Sportsmed 1993;21:111-123.

31 Costill DL, Sherman WM, Fink WJ, Maresh C, Witten M, Miller JM: The role of dietary carbohydrate in muscle glycogen resynthesis after strenuous running. Am J Clin Nutr 1981;34:1831-1836

32 Boisseau N, Delamarche P: Metabolic and hormonal responses to exercise in children and adolescents. Sports Med 2000;30:405422

33 ANC: Apports nutritionnels recommandés (ANC) pour la population française. Paris, Tec \& Doc, 2001

34 Delistraty DA, Reisman EJ, Snipes MG A physiological and nutritional profile of young female figure skaters. J Sports Med Phys Fit 1992;32:149-155.

35 Oppliger RA, Case HS, Horswill CA, Landry GL, Shelter AC: American College of Sports Medicine position stand. Weight loss in wrestlers. Med Sci Sports Exerc 1996;28:ixxii.

36 Moffatt RJ: Dietary status of elite female high school gymnasts: inadequacy of vitamin and mineral intake. J Am Diet Assoc 1984;84 1361-1363.

37 Freischlag J: Weight loss, body composition, and health of high school wrestlers. Phys Sportmed 1984;12:121-126.

38 Constantini NW, Eliakim A, Zigel L, Yaaron M, Falk B: Iron status of highly active adolescents: evidence of depleted iron stores in gymnasts. Int J Sport Nutr Exerc Metab 2000;10:62-70

39 Kirchner EM, Lewis RD, O’Connor PJ: Bone mineral density and dietary intake of female college gymnasts. Med Sci Sports Exerc 1995;27:543-549.

40 Borer KT: The effects of exercise on growth Sports Med 1995;20:375-397.

41 Caine D, Lewis R, O’Connor P, Howe W, Bass $S$ : Does gymnastics training inhibit growth of females? Clin J Sport Med 2001;11:260 270

42 Caine D, Bass S, Daly R: Does elite competition inhibit growth and delay maturation in some gymnasts? Quite possibly. Pediatr Exerc Sci 2003; 15:360-372.

43 Smith WJ, Underwood LE, Clemmons DR Effects of caloric or protein restriction on insulin-like growth factor-I (IGF-I) and IGF-binding proteins in children and adults. J Clin Endocrinol Metab 1995;80:443-449.
44 Theintz GE: Endocrine adaptation to intensive physical training during growth. Clin Endocrinol 1194;41:267-272.

45 Jahreis G, Kauf E, Frohner G, Schmidt HE: Influence of intensive exercise on insulinlike growth factor I, thyroid and steroid hormones in female gymnasts. Growth Regul 1991;1:95-99.

- 46 Bass S, Pearce G, Bradney M, Hendrich E, Delmas PD: Exercise before puberty may confer residual benefits in bone density in adulthood: studies in active prepubertal and retired female gymnasts. J Bone Miner Res 1998; 13:500-507.

47 Daly RM, Rich PA, Klein R, Bass SL: Short stature in competitive prepubertal and early pubertal male gymnasts: the result of selection bias or intense training? J Pediatr 2000; 137:510-516.

48 O'Connor PJ, Lewis RD, Boyd A: Health concerns of artistic women gymnasts. Sports Med 1996;21:321-325.

- 49 Deutz RC, Benardot D, Martin DE, Cody MM: Relationship between energy deficits and body composition in elite female gymnasts and runners. Med Sci Sports Exerc 2000;32:659-668.

50 Dixon G, Eurman P, Stern BE, Schwartz B Rebar RW: Hypothalamic function in amenorrheic runners. Fertil Steril 1984;42:377383

51 Manore MM: Dietary recommendations and athletic menstrual dysfunction. Sports Med 2002;32:887-901.

52 Strauss RH, Lanese RR, Malarkey WB Weight loss in amateur wrestlers and its effect on serum testosterone levels. JAMA 1985;254:3337-3338.

53 Chatard JC, Mujika I, Guy C, Lacour JR: Anaemia and iron deficiency in athletes. Practical recommendations for treatment. Sports Med 1999;27:229-240.

54 Rowland TW: Iron deficiency in the young athlete. Pediatr Clin North Am 1990;37: 1153-1163.

55 Rowland TW, Stagg L, Kelleher JF: Iron deficiency in adolescent girls. Are athletes at increased risk? J Adolesc Health 1991;12: 22-25.

56 Benson JE, Geiger CJ, Eiserman PA, Wardlaw GM: Relationship between nutrient intake, body mass index, menstrual function, and ballet injury. J Am Diet Assoc 1989;89: 58-63.

57 Petrie HJ, Stover EA, Horswill CA: Nutritional concerns for the child and adolescent competitor. Nutrition 2004;20:620-631.

58 Sundgot-Borgen J: Eating disorders, energy intake, training volume, and menstrual function in high-level modern rhythmic gymnasts. Int J Sport Nutr 1996;6:100-109.

59 Kritchevsky D, Weber MM, Buck CL, Klurfeld DM: Calories, fat and cancer. Lipids 1986;21:272-274

-60 Franzoni F, Mataloni E, Femia R, Galetta F Effect of oral potassium supplementation on QT dispersion in anorexia nervosa. Acta Paediatr 2002;91:653-656. 